\title{
CASE SERIES: ANTITUBERCULAR THERAPY-INDUCED HEPATITIS
}

\author{
SIRISHA G* \\ Department of Pharmacology, Kamineni Academy of Medical Sciences and Research Centre, Hyderabad, Telangana, India. \\ Email: dr.sireesha.g@gmail.com
}

Received: 20 March 2020, Revised and Accepted: 22 April 2020

\section{ABSTRACT}

Drug-induced liver injury (DILI) accounts for $20-40 \%$ of cases of fulminant hepatic failure. Antitubercular therapy (ATT) may cause hepatotoxicity which can range from transient asymptomatic rise in liver enzymes to acute liver failure. The drugs in ATT responsible for hepatotoxicity include isoniazid, rifampicin, and pyrazinamide. Case series was done to present three different cases of ATT-induced hepatotoxicity which came to the Clinical Pharmacology and Therapeutics Department in NIMS for management.

Keywords: Hepatotoxicity, Liver enzymes, Management.

(c) 2020 The Authors. Published by Innovare Academic Sciences Pvt Ltd. This is an open access article under the CC BY license (http://creativecommons. org/licenses/by/4. 0/) DOI: http://dx.doi.org/10.22159/ajpcr.2020.v13i7.37525

\section{INTRODUCTION}

Drug-induced liver injury (DILI) is one of the most important reasons for discontinuation of drugs and treatment failures. Antitubercular therapy (ATT) accounts for $58 \%$ of cases of DILI, followed by anti-epileptics.

ATT-induced DILI is suspected when in the absence of symptoms, elevation of transaminases up to 5 times upper limits of normal (ULN) and in the presence of symptoms up to 3 times ULN or twice ULN of bilirubin, provided competing causes such as acute viral hepatitis, autoimmune hepatitis, and other liver diseases that are ruled out [1].

Among the first-line drugs, isoniazid $(\mathrm{H})$, rifampicin $(\mathrm{R})$, and pyrazinamide (Z) have potential for hepatotoxicity, pyrazinamide being the most hepatotoxic followed by isoniazid and rifampicin. DILI pattern can be hepatocellular or cholestatic or autoimmune of infiltrative types. ATT-induced liver damage is hepatocellular type (characterized by predominant elevation of transaminases). ATT-induced DILI is due to metabolic idiosyncrasy due to metabolites released or accumulated during the metabolic process. This may facilitated by genetic factors or polymorphism of drug metabolizing enzymes.

The various risk factors for DILI caused by anti-tubercular drugs include advanced age, female gender, pregnancy, comorbidities such as obesity, diabetes, and underlying liver disease, genetic factors such as acetylator polymorphism, concomitant viral infections such as HIV, hepatitis B and C, and underlying nutritional status such as malnutrition/hypoalbuminemia [2].

\section{CASE NUMBER 1}

A 20-year-old male patient was a known case of Koch's and Gilbert syndrome for 3 years and was on antitubercular treatment. After 3 years of ATT, his total bilirubin increased to $2.6 \mathrm{mg} / \mathrm{dl}$ (normal limits $0.1-0.8 \mathrm{mg} / \mathrm{dl}$ ) and there was increase in conjugated bilirubin to $2 \mathrm{mg} / \mathrm{dl}$ (normal is up to $0.25 \mathrm{mg} / \mathrm{dl}$ ). The case was referred to the Department of Clinical Pharmacology and Therapeutics, NIMS, for antituberculosis (TB) regimen in view of hepatitis.

\section{Advice given}

According to CDC guidelines 2003, isoniazid, rifampicin, and pyrazinamide can all-cause hepatitis that may result in additional liver damage in patients with pre-existing liver disease. However, because of effectiveness of these drugs, they should be used if at all possible, even in the presence of pre-existing liver disease. However, CDC guidelines do not give recommendations with respect to isolated serum bilirubin elevation.

According to CDC guidelines, if AST $>3$ upper normal limits, before initiation of treatment, treatment options are as follows:

a. Rifampicin, pyrazinamide, and ethambutol (RZE) for 6 months RZE

b. Isoniazid, rifampicin, and ethambutol (HRE) for 9 months HRE

c. Rifampicin + Ethambutol for 12 months with fluoroquinolone for the first 2 months

d. $A$ and $B$ regimens are preferable and $\mathrm{C}$ is alternative regimen

e. Frequent clinical and laboratory monitoring should be done to detect DILI.

\section{CASE NUMBER 2}

A 38-year-old female patient is a known case of scleroderma, rheumatoid arthritis, and moderate pulmonary arterial hypertension. She was started on tablet terbinafine for cutaneous fungal infection. The patient was started with empirical ATT 23 days after starting terbinafine. It was seen that serum glutamic oxaloacetic transaminase (SGOT) increased to $245 \mathrm{U} / \mathrm{L}$ (normal 5 to $40 \mathrm{U} / \mathrm{L}$ ) and serum glutamic pyruvic transaminase (SGPT) to $124 \mathrm{U} / \mathrm{L}$ (normal 7-56 U/L) after 2 weeks. The patient was started on liver friendly regimen subsequently, which consisted of tab. ethambutol $800 \mathrm{mg}$, tablet levofloxacin $150 \mathrm{mg}$, and injection streptomycin $750 \mathrm{mg}$ IM daily. She was then referred to the Department of Clinical Pharmacology and Therapeutics, NIMS, for advice.

\section{Advice given}

Among the drugs prescribed, terbinafine, isoniazid, rifampicin, and pyrazinamide are reported to cause hepatotoxicity. In this case, according to CDC guidelines as SGOT/SGPT raised to more than 3 times the ULN with symptoms, isoniazid, rifampicin, and pyrazinamide should be withdrawn.

Rechallenge may be planned, when liver function tests were $<2$ times ULN with one drug at a time under supervision by hospitalization and intense monitoring of liver function tests. Rifampicin increases clearance of terbinafine by $100 \%$. Hence, terbinafine efficacy may be reduced and needs to be avoided when coadministered with rifampicin.

\section{CASE NUMBER 3}

A 31-year-old female patient came with arthralgia and skin lesion, provisional diagnosis of systemic lupus erythematosus was made. She developed seizures and was hospitalized. She had hydrocephalus on computed tomography scan and pulmonary infiltrates were seen on 
chest X-ray and was started on ATT empirically. After 3 weeks, ATTinduced hepatitis was diagnosed with SGOT-320 U/L, SGPT 137 U/L, and total bilirubin/direct bilirubin $3.3 / 3.1 \mathrm{mg} / \mathrm{dl}$. The patient was given injection dexamethasone and subsequently developed acute psychosis. Review opinion was sought regarding modified ATT in view of hepatitis and seizures (as fluoroquinolones reduce seizure threshold).

\section{Advice given}

This is suspected case of central nervous system (CNS) TB with ATT induced hepatitis (SGOT increased to 5 times upper normal limits and increased bilirubin). According to British Thoracic Society guidelines for CNS TB 2009, in such cases, pyrazinamide may be withdrawn, but in this case as bilirubin is elevated, rifampicin also may be stopped. The patient should be started on isoniazid, ethambutol, and amikacin. Liver function tests should be monitored daily and rechallenge be started with rifampicin, once SGOT is $<2$ times the ULN value.

\section{DISCUSSION}

DILI predisposes a patient to develop drug resistant TB. Recurrence of ATT-induced DILI is reported in $10 \%$ of cases [3]. Hence, this ATTinduced DILI should be treated promptly.

Once the diagnosis of DILI due to ATT is established, it is essential to first stop all potentially hepatotoxic drugs till complete clinical and biochemical resolution of hepatotoxicity occurs. In the interim period, at least three non-hepatotoxic drugs such as ethambutol, streptomycin, and quinolones such as levofloxacin or ofloxacin or ciprofloxacin can be used after appropriate evaluation of renal function and visual acuity.
According to CDC guidelines, suspected anti-TB drugs can be started one at a time once the transaminase levels return to $<2$ times the upper normal. Rifampicin is to be restarted first. If the liver functions remain normal after 1 week, isoniazid can be added to the regimen. If the liver functions remain normal after 1 week, then pyrazinamide is added. If there is recurrence of symptoms or deterioration of liver functions, the last added drug should be stopped. Depending on the number of doses taken, bacteriological status, and the severity of the disease, the treatment may have to be individualized and extended [4].

\section{CONCLUSION}

This case series suggest the importance of monitoring of liver function tests during the first 8 weeks of use of antitubercular drugs and also helps the clinician's awareness regarding the treatment modalities to manage such cases of liver injury due to these drugs.

\section{CONFLICTS OF INTEREST}

Nil.

\section{REFERENCES}

1. Ramappa V, Aithal GP. Hepatotoxicity related to anti-tuberculosis drugs: Mechanisms and management. J Clin Exp Hepatol 2013;3:37-49.

2. Devarbhavi H. Antituberculous drug-induced liver injury: Current perspective. Trop Gastroenterol 2011;32:167-74.

3. Natrajan S, Subramaniam P. Evaluation of drug induced liver injury due to anti-tuberculous drugs in directly observed daily therapy. Eur Respir J 2016:48:PA2670

4. Sharma SK, Mohan A. Antituberculosis treatment-induced hepatotoxicity: From bench to bedside. In: Medicine Update. Mumbai: The Association of Physicians of India; 2005. 